ÉGYPTE monde arabe

\section{Égypte/Monde arabe}

$3 \mid 2000$

La censure ou comment la contourner

\title{
Vers une nouvelle jurisprudence islamique
}

Compte rendu et traduction par Mona Akouri

\section{Gamal al-Banna}

Traducteur : Mona Akouri

\section{(2) OpenEdition}

\section{Journals}

Édition électronique

URL : https://journals.openedition.org/ema/811

DOI : 10.4000/ema.811

ISSN : 2090-7273

\section{Éditeur}

CEDEJ - Centre d'études et de documentation économiques juridiques et sociales

\section{Édition imprimée}

Date de publication : 30 juin 2000

Pagination : 203-227

ISBN : 2-87027-856-X

ISSN : $1110-5097$

\section{Référence électronique}

Gamal al-Banna, «Vers une nouvelle jurisprudence islamique », Égypte/Monde arabe [En ligne], 3 | 2000, mis en ligne le 08 juillet 2008, consulté le 07 juillet 2022. URL : http://journals.openedition.org/ ema/811 ; DOI : https://doi.org/10.4000/ema.811

Ce document a été généré automatiquement le 7 juillet 2022

Tous droits réservés 


\title{
Vers une nouvelle jurisprudence islamique
}

\author{
Compte rendu et traduction par Mona Akouri
}

\section{Gamal al-Banna}

Traduction : Mona Akouri

Foi, Loi et discours coranique (Nahwa Figh-in Gadîd, vol. 1)

1 Deux parties principales se partagent ce premier volume de la trilogie dont le projet est de jeter les bases ou sources fondatrices (usûl) nouvelles de la jurisprudence islamique (fiqh).

Approches et notions

2 La définition d'une méthode d'appréhension du domaine religieux en général que nous offre cette première partie nous place au cœur du débat actuel sur le rapport à la religion.

3 L'innocence originelle. Le premier chapitre traite de "L'innocence originelle et [du] cercle du licite et de l'illicite ». Le principe fondamental d'abord mis en relief est celui selon lequel en islam, à l'origine des choses est le licite (halâl), l'autorisé, et que seul est illicite (harâm) ce que Dieu a interdit dans son Coran par un verset explicite n'admettant de ce fait aucune interprétation - ou dans un hadith dûment établi et reconnu authentique de la tradition du Prophète (sunna). Il est vrai que les livres de jurisprudence reproduisent cette vérité, mais de manière mécanique, sans réelle conscience de la profondeur et de la globalité de son contenu, preuve en est qu'il n'a guère été donné au principe de "l'innocence originelle » la place qu'il méritait parmi les fondements du figh alors qu'il « dépasse largement la théorie du Droit naturel » ainsi que d'autres fondements du Droit. Un tel principe, estime l'auteur, restreint évidemment les compétences du législateur " de métier " (musharric) et du faqîh, enclins à poser des interdits. Il rappelle dans ce contexte le débat historique, tout à fait «stérile, engagé sur la question de l'acte autorisé (mubâh) censé faire l'objet d'un statut légal » et stigmatise l'attitude des fuqahâ' qui intervertit l'ordre des choses, « jetant le musulman dans l'hésitation devant toute action en l'amenant à se demander si ce qu'il va faire appartient à la catégorie de l'illicite ou à celle du licite ». 
Rappelant le refus des mutazilites de codifier « l'autorisé » au motif que le non-délit ne peut avoir un rapport autre que négatif à la loi (sharc, sharîa), l'auteur conclut qu'en substituant la classification minutieuse des interdits et des actes autorisés - ainsi que la gamme des nuances qui déterminent l'action - à l'appel à la clémence (cafw), pratique ô combien consacrée par le Coran et la sunna, qu'en oubliant le fondement de l'innocence et ses implications, le législateur de métier révèle son désir de contrôler tous les faits et gestes de l'individu, de se servir du moindre prétexte pour assiéger sa liberté.

5 Foi et Loi. À ce stade, l'auteur procède à une mise au point terminologique. Celle-ci révèle des confusions formées au long des siècles et des pratiques pouvant mener à des abus qui ont tôt fait de mettre en évidence des enjeux de pouvoir. Ainsi la définition du mot sharia (Loi divine) est loin de faire l'unanimité chez les savants. Ils sont légion à considérer qu'elle englobe aussi bien les normes religieuses relatives à la croyance, à la foi que les normes sociales, juridiques, etc. L'auteur estime quant à lui, et en accord sur ce point avec l'imam Mahmûd Shaltût (recteur d'al-Azhar dans les années cinquante), qu'il est vital de faire une distinction entre ce qui a trait au domaine de la foi et des croyances et ce qui relève de la Loi proprement dite (sharia), soit entre le principe fondamental (asl) et ce qui en est dérivé, ce qui en est une conséquence (far); il souligne la différence de nature entre la croyance et la Loi : la première a pour siège le cœur et pour pivot la foi, elle concerne l'individu, s'exprime dans le culte (ibâdât) et seul Dieu en est juge, alors que la Loi a pour siège la raison, pour axe et but la justice, concerne le groupe social et ses comportements, ses relations à l'État, la vie économique, politique, etc. (mucâmalât) et relève de l'autorité d'un gouvernement. Il y a parfois interférence entre les deux domaines comme dans le cas de l'aumône légale purificatrice (zakât).

6 Cependant foi et Loi forment un couple indissociable, mais s'il est important de les distinguer, c'est pour éviter de tomber dans des pratiques autoritaristes pour tout ce qui touche à la foi car, comme l'affirme Gamâl al-Bannâ, «la liberté de croyance en islam » est une priorité. Ceux qui appellent à l'application de la sharia par n'importe quel moyen en oubliant que celle-ci ne peut s'exercer et se nourrir que dans un climat où règne la foi, prouvent qu'ils n'ont en tête que le pouvoir (sultân), alors que le concept de gouvernement en islam (hukm) s'apparente davantage à la justice et à l'arbitrage.

D'autre part, au sens le plus large, le mot figh signifie connaissance, compréhension exhaustive. Celle-ci doit être relative aussi bien au domaine de la foi qu'à celui de la Loi. [C'est pour l'interprétation de la Loi que la traduction du mot figh par jurisprudence nous paraît moins inadapté]. Or les fuqahâ ' ont dissocié le figh de la caqîia, l'ont formalisé en l'amputant de la méditation et en le limitant à l'expression du culte (cibâdât) et de la sharia, et ont transféré au compte de la théologie (cilm al-kalâm) tout ce qui avait rapport aux croyances. Ce fut « une erreur fatale », explique l'auteur, car « la foi est la clef de voûte de toutes les religions » et, plus généralement, constitue la base nécessaire de toute éthique. L'autre " erreur fatale » fut d'avoir introduit la question de l'imamat ou khilâfa (gouvernement) dans le registre de la foi alors qu'elle devrait constituer un des fondements de la sharia.

Évolution historique du fiqh cultuel. L'auteur analyse ensuite le processus historique qui mène à l'apparition puis à l'inflation du figh relatif au culte (figh al-cibâdât). Le culte occupe une place privilégiée dans toutes les religions puisqu'il est l'expression du lien entre l'homme et Dieu et distingue la religion des autres croyances, mouvements d'idées ou systèmes, tels que la philosophie, la politique, etc. Avec l'apparition des 
religions révélées, le culte n'a pu, malgré la lutte en ce sens des prophètes, se libérer d'un héritage plusieurs fois millénaire de pratiques païennes; pratiques qui emprisonnent la configuration de Dieu dans un ensemble de projections humaines semblant, avec le temps, faire partie intégrante de la religion - ce qui historiquement a ouvert la voie à toutes les manipulations effectuées par la caste des puissants et des prêtres.

9 À l'époque du Prophète, le culte était toujours associé à l'action et l'intention primait le rituel et la forme car en islam, souligne l'auteur, «le culte ne peut vraiment polir les âmes qu'au cas où la société se trouve libre de l'oppression et de la corruption ». Avec la conquête et la fulgurante expansion du territoire de l'islam, la complexité croissante exige une compréhension et une interprétation au moins aussi complexes que la nouvelle réalité et donc une spécialisation.

Le figh naît, s'organise en harmonie avec le califat. Lorsque le pouvoir passe aux mains des «bâtisseurs d'empires » (les dynasties Omeyyade, Abbasside, etc.), influencés par le mode de gouvernement romain aux dépens de celui des conseils consultatifs (shûra), les fuqahâ' s'opposent dans un premier temps au calife - parvenant même à détrôner alWalîd bin Yazîd -, mais ces procédés révolutionnaires cèdent rapidement le pas à une résistance passive et à « un rapport dialectique » au pouvoir, entretenu sous le signe de la contradiction, «donnant la mesure de la complexité et du paradoxe qui gouvernent les relations sociales et en font tout ce qu'il y a de plus éloigné de la logique.» Ainsi renonce-t-on à des idéaux de justice et de liberté de la pensée au nom de cette même liberté (cf. le comportement des mutazilites, tenants de l'école de la raison - al-caql face à celle des traditionnalistes - al-naql) ou par souci de préserver un califat par l'intermédiaire duquel, malgré tout, l'islam se répandait (Ibn Hanbal et al-Muctasim) but qui s'accommodait de toutes les justifications.

11 Le faqîh perdait de plus en plus de sa légitimité et de son initiative politique, trouvait son champ d'action réduit. Devant cette impasse, une série de facteurs positifs (l'expressivité et l'importance du culte en islam) et négatifs (la restriction des prérogatives du faqîh) poussèrent à la recherche d'une alternative au sein de cette retraite qu'opéra le figh dans les pratiques cultuelles : l'apparition des voies soufies fut l'expression spontanée de masse de cette recherche, bientôt concurrencée par une institutionalisation incarnée par les écoles du figh (madhhab, pl. madhâhib).

12 «Le culte est la synthèse de toutes les actions qui plaisent à Dieu » : cette définition pertinente ainsi que d'autres proposées par l'auteur n'en demeurent pas moins éloignées de la représentation que l'on se fait communément du culte, le glissement de sens s'étant opéré en faveur d'une forme rituelle, "considérée comme le but premier de la vie »; c'est ce glissement de sens, cette préséance accordée au rite qui devait irrémédiablement aboutir à l'inflation d'un figh cultuel et notamment au phénomène de « fétichisation de la prière » que l'auteur analyse longuement.

13 L'ijtihâd: liberté et restriction. Par ailleurs, le fiqh pose également le problème de l'effort d'interprétation individuel (ijtihâd) dans la mesure où des statuts légaux, des solutions juridiques (hukm, pl. ahkâm)' sont toujours à rechercher concernant les divers aspects de la vie.

14 L'auteur s'en prend d'abord à l'idée répandue selon laquelle il faudrait procéder à « la réouverture des portes de l'ijtihâd» - thèse superficielle qui présuppose que les portes en furent fermées sur ordre du sultan alors qu'historiquement, il s'est plutôt agi d'un 
essoufflement. Si divers pouvoirs ont quelquefois joué un rôle restrictif, ce fut après que les ulémas eurent mis leurs savoirs et méthodes par écrit, soucieux en cela d'enrayer l'anarchie des références à laquelle un recours à une sunna encore mal connue avait abouti chez les fuqahâ".

Or, si l'on veut restaurer l'ijtihâd dans sa vigueur première, il faut, estime l'auteur, déterminer de nouveaux fondements (usûl) pour le figh, des fondements susceptibles de se mesurer à l'évolution historique et sociale : ce dernier, en effet, ne peut continuer à fonctionner selon des règles édictées il y a mille ans. Pour ce faire, il préconise la création d'un conseil consultatif et pluridisciplinaire permanent, composé de fuqahâ' et autres spécialistes, car un seul individu ne peut prétendre être versé dans tous les domaines et « si l'esprit de la sharia est le même, il existe néanmoins une différence de nature entre les solutions juridiques. » Cela permettrait, en outre, au fiqh, «notamment en matière de règles et de codes de procédure pénale, de s'ouvrir sur toutes les expériences humaines... »

16 Cependant, il ne faudra s'attendre à "cueillir les fruits " des avis de ce conseil ou d'autres initiatives qu'une fois instituée une nouvelle méthode et «déterminés les nouveaux fondements du figh» - lorsqu'en particulier, et comme l'a préconisé l'imam al-Ghazâlî, «la raison comme source » se sera substituée au raisonnement analogique « qui ne peut constituer une source ». Alors seulement permettra-t-on à l'ijtihâd d'être non plus un moyen de déduire, de faire apparaitre (muzhir) à partir d'indices probants (dalîl, pl. adilla) trouvés dans les textes, des statuts légaux, mais d'élaborer directement ces statuts, de les construire (munshi') en s'appuyant sur « l'esprit du texte ».

La compréhension du discours coranique

Dans l'introduction à ce premier volume, l'auteur annonçait, à titre programmatique, les nouveaux fondements du figh qu'il se propose d'établir dans le deuxième volume et précise que ces derniers seront ceux de la Loi (usûl al-sharîa), conformément à la distinction faite entre foi et Loi, caqîda et sharî́a. Au nombre de quatre, ces derniers s'énumèrent comme suit: la raison ( $a l-c a q l)$; les préceptes juridiques et principes moraux tirés du Coran (al-ahqâm wa-l-qiyam al-qur'âniyya) ; la tradition du Prophète (alsunna); et la coutume (al-curf), entendu ici au sens de droit coutumier. Si la raison semble avoir pris la première place, celle dévolue au Coran dans les fondements du figh (usûl al-figh), c'est qu'elle est justement une condition nécessaire à la compréhension du Coran et de ses préceptes.

18 Le Coran comme fondement de la foi (asl al-caqîda), sans laquelle la mise en pratique de la Loi ne saurait être justifiée, est donc le principe premier à partir duquel tout commence, principe qui procède à la «re-création des âmes» et c'est pourquoi une part importante de l'ouvrage est consacrée à la manière dont le " discours coranique » prépare à une compréhension maximale des préceptes divins : cette dernière ne devrait donc rien aux exégètes ni aux hommes de lettres ou à divers penseurs cherchant à en dévoiler la secrète rhétorique.

19 L'époque du Prophète où, «à elle seule, la récitation du texte sacré suffisait à amener les associationnistes à la foi »- de sorte qu'on a pu dire que «la Conquête de Médine s'était faite par le Coran »-, était une époque privilégiée. Les livres d'exégèse n'avaient pas encore donné la chasse au Livre. L'auteur rapporte cet avertissement du Prophète concernant le Coran: "Si une explication fait mouche, elle n'en a pas moins raté la cible ", formule d'une extrême concision qui dit combien sont multiples les facettes du Coran, de sorte que ne peut être favorisée une interprétation au détriment d'une autre, 
ni une idée unique, de l'hégémonie de laquelle il entend en tout cas libérer l'esprit. Toutefois, lorsque Médine se transforma pour devenir un centre rayonnant du monde, et qu'eurent éclos les grandes villes de l'islam, Bassora, Kûfa, Fustat, Kerouan, «les Arabes devinrent une minorité et les compagnons du Prophète une monnaie rare ", et l'apparition de l'exégèse se trouva une justification dans «la soif de savoir» des populations nouvelles.

20 L'obstacle exégétique. Bientôt, les célèbres exégèses d'al-Tabarî, al-Qurtubî, Ibn Kathîr, Ibn 'Atiyya, al-Zamakhsharî, etc., pourtant saturées de fables et d'historicismes (asbâb al-nuzûl), acquirent un halo de sacralité tel « qu'il fut impossible à Ibn Taymiyya, Ibn al-Qayyim ou Ibn Hazm de rien changer » malgré leurs travaux de décantation.

Les repères historiques que donne l'auteur permettent de se faire une idée précise de la science exégétique, de la révolution accomplie par Muhammad cAbduh dans ce domaine et englobent aussi bien les approches modernes que contemporaines du Coran, longuement exposées et commentées, celles notamment: de l'islamologue Mohammed Arkoun - à qui l'auteur reproche de n'avoir "pas su se libérer de la manière dont l'anthropologie européenne pense la religion »; du penseur syrien Muhammad Shahrûr - dont l'auteur salue « le considérable et sincère effort pour arracher la pensée arabe et la jurisprudence islamique à leur étouffante crise et tenter d'élaborer une méthode de lecture objective du Livre de Dieu » mais pour aussitôt juger exagérément linguistique cette dernière ; celle, enfin, de Nasr Hâmid Abû Zayd «chez qui se sont mêlés le pire produit de l'orientalisme et ce que notre tradition a donné de plus mauvais. " [Cette dernière critique ne doit pas faire oublier la position de l'auteur qui, fidèle à son militantisme pour la liberté de la pensée, s'était fermement élevé contre le procès pour apostasie intenté à Abû Zayd - ce qu'il rappelle d'ailleurs lui-même dans une longue note.] Parallèlement aux diverses interprétations du texte coranique ont été déployés des efforts innombrables pour saisir les secrets du miracle, du prodige coranique (aligâaz). Leur échec tient, selon Gamâl al-Bannâ, au fait que les auteurs de tels ouvrages «n'ont guère lié la forme du message au but pour lequel il fut révélé... »

Le Coran : miracle de l'islam. "La compréhension du Coran telle qu'elle doit être " clôt cette seconde partie du présent volume. Il y est dit en substance que le Coran est le miracle de l'islam - un prodige constamment manifesté et renouvelé dans l'âme humaine. D'où il apparaît que l'islam a transféré le miracle du champ des lois naturelles à celui de l'âme humaine parce que c'est en elle qu'il peut indéfiniment accomplir son prodige, en garantir la pérennité pour tous les temps. Telle est « la mission impossible » dont s'acquitte le Coran.

L'auteur y analyse ensuite les «signes » du prodige coranique : sa structure musicale qui, imprégnant l'ouïe, s'empare de la totalité de l'être ; "les figures du discours »; la mobilisation de toutes les formes d'esthétique du langage ouvrant au lecteur l'accès au sens en dépit des obstacles dus à l'insuffisance de la langue et du caractère limité de l'entendement humain - notamment pour ce qui est de concevoir l'Être de Dieu et la vie à venir; l'approche toute psychologique de l'homme, pénétrant les plus délicates subtilités de l'âme, touchant ses cordes les plus sensibles pour enfin assumer l'orientation de l'être humain qui, entré en parfaite interaction avec le Coran, se trouve guidé vers deux principaux objectifs, en fait deux exigences: l'usage de la raison et celui de la foi, dans les préceptes et principes moraux fixés par le texte sacré, condition d'» une nouvelle venue au monde de l'homme engendré par la foi. »

La sunna (Nahwa figh-in gadîd : al-sunna, vol. 2) 

prophète Muhammad), constitue le matériau de la jurisprudence islamique en ce qu'elle concerne les détails de la vie quotidienne alors que le Coran vise l'absolu et l'universel. D'où sa primauté comme source de législation (masdar tashrî́) qui doit éclairer, justifier et mettre en pratique les préceptes généraux du Coran dont elle se nourrit. Il est important de noter la différence soulignée par l'auteur entre la sunna comprise dans son sens originel (l'action fondatrice du Prophète, sa ligne de conduite synthétisante, générée par les principes coraniques qui lui donnent son sens exhaustif), et la sunna entendue simplement au sens de hadith ("paroles rapportées »), étant donné que les deux notions « sont aussi éloignées l'une de l'autre que la parole l'est de l'action » et que, par ailleurs, elles réfèrent également à des étapes distinctes de la législation islamique, de par les acteurs comme de par les pratiques normatives.

La rigoureuse interdiction du Prophète concernant la transcription de ses paroles est un autre point important à retenir. Ainsi, les premiers califes s'étaient inclinés, détruisant ce qu'ils avaient consigné pour éviter, selon le célèbre mot de 'Umar, « qu'un autre livre ne vienne faire oublier le Livre de Dieu ». Mais, avec le temps, une véritable problématique s'installe, surtout avec l'extension du territoire de l'islam, la raréfaction puis la disparition des compagnons (sahâbî, pl. sahâba) dépositaires de la Tradition vécue, qui avaient vu le Prophète agir et l'avaient entendu donner des avis juridiques (fatwa ou futya), et la nécessité se fait sentir d'une législation susceptible de gérer les intérêts des gens dans des sociétés autrement plus complexes et diversifiées : l'oralité, non plus que l'exemple des habitants de Médine, ne pouvaient plus suffire à transmettre la Tradition active et pratique (al-sunna al-ficliyya wa-l-amaliyya). Au début du premier siècle de l'hégire, de plus en plus d'arguments étaient donc réunis afin d'entreprendre une collecte systématique et une transcription du hadith (tadwîn alsunna), ce qui devait durer jusque vers le milieu du IVe siècle de l'hégire, période qui fut appelée "ère de la codification " (asr al-tadwîn) et qui consacrait le grand mouvement de collecte et de transcription du hadith et de l'expérience juridique accumulée depuis l'époque du Prophète. Un consensus s'était donc formé pour contourner l'interdiction initiale tout en fixant des règles de codification censées être assez strictes pour respecter l'intention et la volonté de ne point créer d'entité, de référence morale, qui puisse rivaliser avec le Coran.

La sunna dans la jurisprudence des pieux ancêtres (al-figh al-salafí)

La première partie de l'ouvrage nous emmène au cœur du savoir réuni par les anciens L'auteur procède à un passionnant inventaire critique, alternant l'histoire de la constitution du corpus de la sunna, entendue dans le sens de hadith (al-sunna alqawliyya), et la constatation d'un état de fait inscrit dans la longue durée, de moins en moins susceptible de répondre aux impératifs spirituels et pratiques. Et ce faisant, il restitue dans ses divers contextes cette problématique résurgente des origines de la sunna/hadith et des règles de transcription établies par les traditionnistes (muhaddith, pl. muhaddithûn).

27 Les divers développements d'ordre subjectif (exploitant l'ascendant du Prophète sur les cœurs au point que, par bonne ou mauvaise intention, furent transmises des " paroles » qui n'étaient pas les siennes) ou objectif (le besoin pressant d'un référent juridique puis la responsabilité de gouvernants favorisant l'inflation du figh cultuel et l'apparition de hadiths partisans, relatifs aux califats omeyyade ou abbasside et dont certains «devinrent des piliers du figh chiite ») ont fait de la sunna/hadith une concurrente du 
Coran sur lequel, dans l'usage courant, elle a fini par prendre le dessus. La consécration de cet état n'était plus qu'une affaire de temps. L'auteur en distingue les étapes.

Création d'une science. "Vers la moitié du II siècle de l'hégire, le monde musulman était submergé par une marée de près d'un million de hadiths " qu'on se résolut à "passer au tamis »; l'opération présidait ainsi à la création d'une science nouvelle et des institutions qu'elle suppose. Deux tournants décisifs devaient en marquer l'orientation et l'évolution : le premier fait suite à la composition, par l'imam al-Shâficî, d'un traité (al-Risâla) dans lequel il déduit les fondements du fiqh en donnant au hadith une place prépondérante. Le second était pris au $\mathrm{V}^{2}$ siècle $\mathrm{H}$, « lorsque les quatre grands madhhab en se confortant, se rallièrent tous - malgré leurs divergences - sous la même enseigne", partant du principe que "tel hadith, s'il est authentique, constitue mon école (fa huwa madhabî)».

Si ce "passage au tamis " contribua de manière considérable à réduire le nombre de hadiths transcrits dans les codes, il n'en demeure pas moins que «son impact fut beaucoup plus d'ordre quantitatif que qualitatif » : au bout du compte, en effet, ce sont les hadiths les plus imbibés de négativisme, de bigoterie et d'imitation servile qui l'emportèrent, contribuant à former cet « état d'esprit typique » que caractérisent «le manque de résistance politique, l'occultation du rôle social de la femme, le défaut de pensée esthétique...»

Critiques et partisans. À chaque époque ses enjeux. À travers l'Histoire toutefois, la sunna/hadith a suscité avec constance un éventail de réactions. L'auteur en indique quelques-unes qui font date, allant de la "critique réservée » à "l'adhésion sans réserve ». Les points de vue critiques sont diversifiés et proviennent historiquement de camps souvent opposés. Ainsi en est-il déjà, bien avant l'ère de la codification, des Khârijites et des Chiites. Les premiers avaient remis en cause des hadiths, dont un notamment qui fixait le choix de l'imam parmi la tribu de Quraysh. Un moment regroupés autour de l'imam Jafar al-Sâdiq, "que l'on peut considérer comme le véritable fondateur du figh et de la politique chiites", les seconds (" cet autre produit de la grande sédition" quoique "politiquement plus habiles» que les premiers), avaient émis des réserves si importantes relativement aux sources, en particulier, qu'ils finirent par créer leur propre chaîne de transmission du hadith remontant jusqu'au Prophète (isnâd). Les mutazilites, quant à eux, qui se virent reprocher par les traditionnistes d'avoir introduit l'esprit d'examen (caql) « là où il aurait fallu selon eux, se contenter de reproduire (naql) sans raisonner ", refusèrent, à l'instar des Chiites, de considérer dignes de foi tous les compagnons du Prophète (sahaba). Ce questionnement rationnel devait se retrouver chez Ibn Khaldûn qui, davantage préoccupé par le contenu (matn) que par les modalités de transmission (isnâd), l'intégra à sa méthode de lecture de l'Histoire et ne manqua pas d'interroger la science du hadith et celle de l'exégèse (tafsir), montrant que tout texte qui ne résistait pas à l'examen de la raison risquait de «perdre de sa crédibilité. »

31 La pensée contemporaine critique à l'égard de la sunna/hadith (Tawfîq Sidqî, Ahmad Amîn, Mahmûd Abû Rayya et tout récemment Ismaîl Mansûr)', en butte aux grands bouleversements de l'époque et soucieuse, soit de donner des bases plus solides à la pensée religieuse, soit de prétendre prouver la caducité de celleci devant « l'irrésistible ascension » du progrès, mettait à nouveau en jeu la question de la codification de la sunna en rappelant la franche interdiction du Prophète comme elle mettait à l'épreuve 
les datations attestées - ce qui malgré tout amenait à s'intéresser à la teneur des textes et à sonder les contenus (matn), par delà la seule question de l'isnâd.

Quant aux ouvrages des partisans de la sunna, l'auteur indique qu'ils sont tout simplement innombrables. Exemplaire, la Risâla de l'imam al-Shâfici est citée à nouveau pour son rôle dans la préséance accordée à la sunna dans l'explicitation - bayân - du Coran. Exemplaire également l'ouvrage, ancien, de l'uléma Ibrâhîm Ibn al-Wazîr', aveuglément apologétique à l'égard des sahaba, de leurs compagnons (tâbici) et de toute personne ayant vécu au temps du Prophète sans même l'avoir connu. Pur échantillon de la scolastique dans laquelle, estime l'auteur, se sont noyés les fuqahâ' et à leur suite les muhaddithûn, où l'on voit à l'œuvre "une argumentation truffée de sophismes, empruntant à la manière surannée des théologiens grecs». Exemplaire enfin, à l'époque contemporaine, l'ouvrage du cheikh Mustafâ al-Sibâ î́: écrit dans le sillage de la polémique suscitée par les critiques peu pertinentes de cheikh Mahmûd Abû Rayya dans son ouvrage Lumières sur la sunna de Muhammad (1950), il y répond violemment; défend la sunna "qui atteste et conforte la plupart des statuts juridiques du Coran " pour mieux contredire les thèses d'Ahmad Amîn et de 'Alî Hasan 'Abd al-Qâdir'; relève les erreurs des Orientalistes; et reproche même à Muhammad 'Abduh de n'avoir pas eu d'égards pour le hadith et d'avoir un temps entraîné Rashîd Ridâ dans cette voie. De style abordable et clair, cet ouvrage n'en exprime pas moins la méfiance à l'égard du raisonnement appliqué au contenu du hadith.

De l'examen des sources à celui du contenu. Reprenant l'argument d'Ibn Khaldûn, Gamâl al-Bannâ invite à chasser cette peur de l'esprit critique «car on fait preuve de bien plus de respect à l'égard de l'Envoyé de Dieu en délestant sa Tradition de toutes les légendes qui en ternissent le sens ". Le problème, indique-t-il, est qu'à aucun niveau on ne s'est soucié d'établir des normes véritablement objectives pour la codification du hadith. Aussi procède-t-il en deux très longs chapitres à un minutieux examen des normes avancées par les fuqahâ' et les traditionnistes. Extrêmement rigoureux dans les formulations initiales d'al-Shâficî, Ibn al-Salâh et Abû Hanîfa, les principes généraux commandant la codification se sont peu à peu relâchés. La raison en serait imputable à la dégradation du climat politique et social survenu dans le territoire de l'islam à partir du IVe siècle de l'hégire mais aussi à la nature des règles édictées par les muhaddithûn afin de développer et « détailler» les grands principes. Parmi ces règles incriminées, citons celles qui se rapportent au traitement des hadiths selon leurs transmetteurs: sanad; aux normes de la réfutation: jarh; au constat de rectitude: tacdîl; et à l'importante question de la classification du savoir : marâtib al-cilm, méthode acquise par la recherche des indices probants (dalîl, pl. adilla) dans l'étude de la sharia. Tributaires de l'interprétation personnelle, ces règles ne pouvaient être appliquées avec précision sans compter que l'identité de leurs usagers était aussi cause de bien des divergences : si les muhaddithûn en effet s'intéressent à la définition du hadith (tacrîf al-hadîth) du point de vue de son degré d'authenticité (darajat al-hadith), les fuqahâa', eux, se préoccupent d'établir en quoi tel hadith constitue ou non une référence probante (hujjiyat al-hadith).

34 Ainsi la brèche était ouverte devant les compromis qui allaient aboutir au laxisme et permettre, "et cela dans les livres les meilleurs", à un hadith apocryphe (saqîm), inventé (mawdîc), de figurer à côté d'un hadith authentique (sahîh), "avec les conséquences désastreuses que cela a pu avoir.» 
dignité dont est entouré le Prophète ne prennent pas leur source dans le seul fait qu'il assure la transmission (tablìgh) du message divin. «Des aspects de sa personnalité et de sa politique sont également mis en avant » et, en commandant l'obéissance à l'Envoyé, le Coran le désigne comme "l'exemple de perfection» (uswatun hasanat) duquel il convient de s'inspirer. C'est ce que s'étaient appliqués à faire les premiers musulmans, du temps où la sunna ne s'était pas encore transformée en hadith, en observant les comportements sociaux, familiaux et cultuels du Prophète (sunna hayâtiyya et sunna cibâdiyya) ainsi que l'acquittement de ses tâches de chef politique (sunna siyâsiyya); comptant, "après le Coran ", sur une inspiration divine (wahî sunni) - cette notion introduite par l'auteur et développée plus loin est capitale pour le figh - et sur l'effort personnel ; et recherchant, dans tous les cas, « l'explicitation du Coran et l'observance de ses préceptes ». Ces "trois sphères de la sunna ", évoluant en synchronie, étaient donc indissociables chez les premiers musulmans pour lesquels, explique l'auteur, l'islam représentait un mode de vie, un appel à la libération et « à la constitution d'une société fondée sur les valeurs du droit, de la justice, de la véracité, de la compassion et de la miséricorde... »

40 Le rôle du chef politique dans ses rapports avec sa communauté revêt donc un aspect privilégié dans la sunna. «C'est cet aspect qui a pratiquement engendré l'Utopie 
politique [au sens de référence et espoir], sous la forme de l'État (dawla) de Médine et des premiers califats ; période qui, pour courte qu'elle fut, n'en a pas moins continué d'inspirer les penseurs islamiques quant aux normes requises pour fonder le régime politique de l'islam.»

En permettant que cette période soit portée au seul compte de l'histoire du leadership, en ignorant son statut de sunna constitutionnelle, son aspect pilote, initiatique et ce qu'il suppose en termes d'obligation pratique, la société musulmane se condamnait à la régression «en perdant ses deux armes les plus précieuses : l'idée de Dieu, fondement de la foi (dénaturée par la substitution de la logique de la théologie à la logique du Coran); et l'idée d'une responsabilité politique, assumée par un chef initié aux valeurs coraniques de justice, de liberté, etc., illustrée par l'exemple de l'Envoyé, fondement de la sunna et donc de la Loi ». Comment ne pas en être convaincu, s'interroge l'auteur, lorsque l'on assiste à la "tragédie » des dictatures contemporaines «qui atteint son paroxysme en Afghanistan ». De plus, ajoute-t-il, l'ignorance ou l'abandon des bases législatives, politiques et financières de gouvernement, instaurées par le Prophète, ont privé les révolutions modernes, notamment française et bolchévique, d'une référence précieuse qui aurait peut-être pu empêcher que « la violence sanguinaire » ne devienne " une tradition révolutionnaire légitimée » et incontournable".

En conclusion, la question de l'imamat (khilâfat ou hukm) - déjà évoquée dans le premier volume - a doublement été lésée : d'une part parce que, pour des raisons historiquement connues, la tradition chiite l'a exagérément montée en joyau de la foi, et, d'autre part, parce que les fuqahâ' ne lui ont guère reconnu son statut fondamental dans la Loi. Or, indique-t-il, « considérer la question du gouvernement dans le cadre de la Loi lui confère la sacralité de l'islam [valeurs ou pratiques] sans l'ériger en théocratie... ", dans la mesure où le Prophète a institué la souveraineté de la Loi ; et «si la sharia sanctionne le voleur ou l'adultère, comment ne demanderait-elle pas des comptes au détenteur du pouvoir qui trace les politiques et se porte garant de la Loi elle-même?»

Entre Coran et sunna : les deux niveaux de la révélation. Les limites assignées dans certains versets à la mission du Prophète - réduite à sa seule fonction de transmetteur (muballigh) - doivent se comprendre dans leur contexte exact : il s'agissait de faire en sorte que l'autorité conférée ne se mue pas en un pouvoir coercitif. C'est ce qui a échappé à un faqîh de la carrure d'Ibn Hazm, toutefois opposé en bien des verdicts aux jurisconsultes de son temps en raison de son amour de la justice et de l'égalité, mais qui a vu dans ces versets une limitation de la sunna au seul cadre de la parole (al-sunna alqawliyya).

44 Ayant fait remarquer que «la transmission (balâgh) du message divin n'est pas mécanique puisque le Coran lui donne pour corollaire l'explicitation (bayân)», l'auteur revient dans un chapitre entier sur les questions associées de la transmission et de l'explicitation (al-balâgh al-mubîn) pour en arriver à l'interrogation cruciale concernant ce qui dans la sunna/hadith a oui ou non force de loi (tashrî́ aw lâ tashrî́). D'où il apparaît, en premier lieu, que le Coran s'est globalement limité aux principes généraux (offrant ainsi une marge de flexibilité susceptible de ménager une adaptation à tous les temps); et, en second lieu, que l'Envoyé a assumé l'explicitation de ces préceptes, explicitation soutenue par une inspiration divine (wahî sunnî) moins systématique que la révélation coranique (wahî qur'âni) et différente d'elle « dans la forme comme dans le fond », ne pouvant donc être confondue avec elle en ce qu'elle ne constitue nullement 
le même degré d'obligation que le Coran: celui-ci, note l'auteur, ne manque pas d'intégrer, quand il le faut, des détails explicatifs « comme il en est par exemple dans le verset le plus long relatif à l'endettement ».

La notion de wahî sunnî développée par l'auteur est capitale dans la mesure où elle subordonne l'autorité de la sunna/hadith à celle du Coran alors que la tradition jurisprudentielle en fait au moins l'égale du Coran - allant jusqu'à lui reconnaître une autonomie - notamment depuis qu'al-Shâficî avait systématisé la fonction éclairante (bayân) de la sunna dans sa Risâla, rendant l'interprétation du Coran dépendante de celle-ci. Cette nouvelle notion introduite par l'auteur pourrait contribuer à résoudre la crise du figh en brisant son immobilisme et en l'amenant à gérer librement et efficacement les problèmes relatifs aux besoins de l'époque.

Dans ce contexte, indique-t-il, l'interdiction de la transcription de la sunna apparaît sous un jour nouveau si l'on considère, d'une part, l'aspect universaliste du Coran et, d'autre part, les dits du Prophète, des premiers califes et des compagnons qui convergent pour témoigner de la temporalité de la sunna qui ne peut prétendre à la pérennité du Coran, notamment en ce qui concerne les statuts légaux (ahkâm), « même si des générations s'y sont conformées, car de nouveaux facteurs surviennent » qui nécessitent un effort d'interprétation. Celui-ci est le bienvenu, estime l'auteur, « même s'il contredit les statuts du hadith, à condition d'être en accord avec les principes immuables du Coran. »Si donc les qualités et valeurs incarnées par le Prophète ont indiscutablement un caractère de permanence et supposent l'adhésion morale et pratique, il ne peut en être de même pour des statuts ou solutions juridiques - et même pour certains aspects du culte -, soumis au rapport de l'espace et du temps, exceptés ceux qu'appuie un texte coranique explicite".

À ce niveau, l'auteur expose le point de vue des fuqahâ' (et les débats contemporains) sur ce qui dans la sunna/hadith a force de loi. Il y distingue deux perspectives :

- la perspective référentielle (al-Shâtibî), où les statuts légaux sont attestés par la recherche d'indices probants (dalîl) catégoriques et définitifs (qatci) ou non reconnus pour tels lorsque l'indice est hypothétique, conjecturel (zannî);

- la perspective fonctionnelle, relative au fond, où sont analysés les divers niveaux des comportements du Prophète et tout ce qui émane de lui (Ibn Qutayba, al-Qarâfî, Shaltût, Shâh Walî-Allâh de Delhi).

En annexe à ce chapitre, l'auteur cite l'opinion de Rashîd Ridâ concernant la transcription de la sunna, d'où il ressort que les compagnons se sont pliés à l'interdiction du Prophète " parce qu'ils n'ont pas voulu instituer le hadith en religion à l'instar du Coran ", comme il reproduit la récente fatwa d'al-Azhar, [datant des années quatre-vingt et très significative], où il est reconnu que « l'indépendance » de la sunna à l'égard du Coran « en ce qui concerne les décrets d'obligation et de prohibition... ne fait pas l'objet d'un consensus parmi les ulémas » et ne peut donc être considérée comme un fait indéniable de la religion.

L'arbitrage du Coran. Si donc l'autorité primordiale du Coran se lit d'abord dans le Coran même (Dieu est l'unique Législateur, c'est Lui l'ultime recours et le juge) comme dans la Tradition du Prophète, la pratique des compagnons et celle des premiers califes, la sunna/hadith doit alors être relue suivant une norme qui ne peut procéder des règles de classification et d'authentification des muhaddithûn, sur lesquels l'auteur revient, complétant le diagnostic élaboré dans la première partie du présent volume (chapitres 
3 et 4). Soucieux d'échapper "au piétinement et à la tendance à l'éclectisme » qu'anciens et modernes n'ont pu éviter tant est grande "la fascination » du hadith (futnatu al-hadith), l'auteur propose une norme de lecture dont l'objectivité procède non pas de ce qu'elle reconnaît l'ascendant du Coran " car ceci est une évidence » - restée trop souvent formelle et aléatoire toutefois parce que non systématisée -, mais de ce qu'elle se donne le Coran comme grille permettant "une mesure méthodique ", une sorte de tri du hadith au moyen duquel on reconnaîtra ce qui concorde ou ne concorde pas « avec le texte même des versets ou avec les principes moraux qui s'en dégagent et constituent l'esprit de l'islam ».

Ce faisant, l'auteur procède à une classification par la négative, si l'on peut dire, dans la mesure où des catégories de hadiths sont remises en cause parce que, selon le point de vue développé plus haut, elles ne correspondent pas à l'esprit du Coran ou pour des raisons d'ordre temporel. Ainsi en est-il :

- $1^{\circ}$ ) des hadiths relatifs au mystère du monde invisible (al-ghayb) englobant la mort, le paradis, l'enfer, etc., parce que « Le mystère des cieux et de la terre n'appartient qu'à Dieu » (Cor. 16, 77) ;

- $2^{\circ}$ ) de ceux qui explicitent des passages imprécis du Coran, en invalident des versets, les abrogent, en ajoutent d'autres ou évoquent les circonstances de la Révélation (asbâb alnuzûl);

- $3^{\circ}$ ) de ceux qui contredisent les impératifs coraniques, en particulier la justice ;

- $4^{\circ}$ ) de nombreux hadiths qui déprécient la femme comme ceux concernant une époque révolue et qui sont relatifs au mariage ou au divorce, au statut des esclaves, au tribut, au butin, etc.;

- $\left.5^{\circ}\right)$ de ceux qui attribuent des miracles au Prophète, sachant qu'ils contredisent la centralité du Coran, seul miracle de l'islam;

- $6^{\circ}$ ) de ceux qui privilégient des individus, des contrées, des races, des wâli (détenteurs du pouvoir) ou des califes, etc., le Coran privilégiant la piété et l'action ;

- $7^{\circ}$ ) de ceux qui contredisent les nombreux versets relatifs à la liberté de croyance ;

- $8^{\circ}$ ) ceux qui menacent des pires châtiments ceux qui commettent d'infimes erreurs ou s'adonnent aux simples plaisirs de la vie ;

- $9^{\circ}$ ) de ceux relatifs aux coutumes alimentaires et vestimentaires qui sont à porter au compte de la chronique historique et ne supposent aucune obligation ;

- $10^{\circ}$ ) de ceux qui recommandent l'obéissance aveugle aux gouvernants indépendamment de leur équité, « parce que l'iniquité (zulm) est dans le Coran synonyme d'impiété (kufr)... »

51 Après ce nouveau passage au tamis à la lumière du Coran, l'auteur est à son tour amené à neutraliser près de deux mille hadiths « dont la moitié se trouverait dans les recueils de Bukhârî et de Muslim (al-sahîhayn) ». À ceux qui pourraient s'en étonner et lui dénier ce droit, l'auteur rappelle qu'au II $^{e}$ et III $^{\text {e }}$ siècle de l'hégire, les grands imams traditionnistes ont retenu pour leurs recueils moins du dixième des hadiths qu'ils avaient entendus, sachant qu'ils se trouvaient devant un phénomène de " prolifération maladive " dû à l'immense appel d'informations manifesté au fur et à mesure que l'on s'éloignait des commencements et qui, simultanément, créait un terrain propice au colportage de hadiths inauthentiques.

Dans ce travail de lecture active et critique, l'auteur voudrait qu'on voie bien « le réel souci de la sunna..., ce mot cher et précieux ", devant être compris dans son sens régénéré de mode de vie qui respecte l'homme et sa liberté, si l'on ne veut pas que «la forteresse du fiqh salafî [qui tire sa légitimité d'un passé sclérosé] » ne s'effondre d'un 
coup comme s'est effondrée la forteresse soviétique, "malgré l'immensité des sacrifices " consentis et du territoire concerné, et dont la chute est certainement imputable au fait qu'elle « avait omis de faire de la liberté sa pierre angulaire ».

Par ce rapprochement qui peut choquer de prime abord, l'auteur ne désire sans doute pas autre chose que de montrer à quel point la religion est l'affaire de l'homme et du présent.

Vers une nouvelle jurisprudence islamique (nahwa figh-in gadid, vol. 3)

Ayant dans les deux premiers volumes jeté les bases préliminaires d'une réflexion nouvelle sur les rapports de la jurisprudence (fiqh) à la foi (caqîida) et à la Loi (sharî‘a) et différencié les trois domaines, puis proposé une compréhension exhaustive du discours coranique qui habilite à affronter la problématique de la sunna et du hadith et à remettre en cause ce qui y contredit l'esprit du Coran, Gamâl al-Bannâ continue dans le présent volume ${ }^{12}$ son examen critique des méthodes de réflexion et pratiques juridiques afin de dégager les perspectives qui permettront d'entrevoir et d'établir les fondements nouveaux de la jurisprudence islamique.

Approches critiques

Les trois niveaux de référence. Dans un long chapitre introductif, l'auteur commence par rappeler le poids du patrimoine jurisprudentiel constitué dans un espace-temps incomparablement plus important que celui dans lequel ont pris place la Révélation et la tradition fondée par le Prophète. Ces trois entités, Coran, sunna et figh, sont données comme autant de niveaux de référence, d'instruments pour la confrontation et l'évaluation des pratiques doctrinales et jurisprudentielles relatives à cinq questions primordiales, la foi (al-îmân) en Dieu, la liberté de croyance (al-ictiqâd), la justice sociale, économique et politique, la condition de la femme et l'esclavage dans sa forme ancienne et sa projection moderne dans le statut des travailleurs".

L'étroite compatibilité des deux premiers niveaux de référence, née de l'intime adhésion du Prophète à l'esprit du Livre, offre le plus souvent un puissant contraste avec le troisième. "En occultant le Coran et la sunna authentique ou en les assujettissant, écrit l'auteur, le fiqh a progressivement pris de la distance par rapport à ses sources premières et s'est forgé des fondements autonomes qui relèvent principalement de la technique professionnelle. »

Si des facteurs d'ordre pratique - tels que l'établissement des textes et la spécialisation -, mais aussi historique, politique et culturel sont à l'origine de cette dérive, ils ne peuvent pour autant la justifier. L'auteur montre qu'en s'éloignant de la logique et de l'esprit du Coran "l'idée de Dieu a été corrompue [par les théologiens] comme l'a été l'objectivité de la philosophie » dont ils ont prétendu se servir, et la liberté de croyance. Cette dernière fut enfermée dans l'aphorisme qui faisait un mécréant - passible de la peine de mort - de "quiconque nie une [vérité] nécessairement déterminée de la religion » (man jahada maclùman min al-dîn bi-l-darûra), « formule dans laquelle tout peut s'engouffrer ». Or, poursuitil, « dès qu'il est porté atteinte à la liberté de pensée, l'idée de justice ne peut être approfondie ». Sur le plan politique et social, «la crainte de la dissidence a érigé la nécessité [du compromis] en vertu »; les fuqahâ’ ont par ailleurs légitimé divers modes d'exploitation des esclaves et des travailleurs et déprécié la condition de la femme.

Le figh entre intérêt, intention et correspondances. L'auteur examine ensuite le rapport des fuqahâ' au texte dans l'exercice de leur métier dès lors qu'il s'agit d'établir 
l'assise de solutions juridiques (ahkâm). Il remarque tout d'abord que la notion de texte ne se limite pas pour eux au Coran ou aux hadiths dûment authentifiés mais les déborde largement pour englober les paroles des compagnons du Prophète (sahaba), des compagnons de ceux-ci (tâbici), etc., et qu'elle s'accommode des libertés prises avec le Coran et la sunna évoquées dans le deuxième volume.

Le concept le plus susceptible de provoquer une polémique dans une tradition jurisprudentielle fondée sur la conformité aux textes (taqlîd) était et reste celui de maslaha (pl. masâlih), mot que l'on traduit par « intérêt ». L'auteur déplore le fait que les fuqahâ' ne se soient pas imprégnés de la terminologie coranique à laquelle fait écho la sunna, où sont déclinés les dérivés du terme salâh (sâlihât, islâh dhât al-bayn), qui connotent la recherche et la pratique du bien, la réconciliation, etc., et desquels procède le mot de maslaha. Synonyme de manfaca (utile, profitable, avantageux), ce dernier s'oppose à mafsada (l'état de corruption, de désordre, de violence). « Ils auraient pu, souligne-t-il, partir de l'intérêt », pris dans le sens de la recherche de ce qui est bon et profitable et non dans celui d'un utilitarisme à la Bentham, « et l'établir comme indice (dalîl) de solutions juridiques». Or, ils ont inversé les choses et occulté le problème en considérant que le Législateur (Dieu) était mieux à même de définir l'intérêt des humains, qu'ils ne pouvaient le concurrencer sur ce point, que la sharia avait pour intention (maqsad, pl. maqâsid) le bien-être des gens et qu'il n'existait aucun intérêt qui ne fût déjà défini par les textes. On pourrait penser que les fuqahâ' s'étaient ménagé une marge de manœuvre avec ce qu'ils appellent les intérêts non mentionnés (al-masâlih al-mursala) mais en fait les hésitations et réserves l'ont emporté et l'expression d'une préférence juridique (istihsân) comme la recherche de l'intérêt de la communauté (istislâh) ont été considérés, notamment par al-Shâficî, comme une entorse au droit du Législateur.

60 Les fuqahâ' se sont donc cantonnés dans le négativisme en travaillant à définir les tentations corruptrices et à les bannir (sadd al-dharẩic, daf al-mafsada). "En réalité, ils ignoraient le texte en prétendant s'incliner devant ses normes. »

Il a fallu « le cri d'al-Tûfî (m. 716 H) qui proclamait la priorité de l'intérêt sur le texte » pour remettre en mémoire les prérogatives des califes 'Umar et cAlî qui allaient au devant des besoins sans céder à la hantise du texte. L'auteur cite nombre de fuqahâ' modernes et contemporains qui ont eu à cœur, autant que les anciens, de combattre la thèse de Najm al-Dîn al-Tûfî. Celui-ci pourtant ne dérogeait pas qualitativement à la règle de la jurisprudence traditionnelle et avait pris le soin de préciser que, correctement interprété, un texte ne pouvait contredire l'intérêt général; il s'était en outre armé d'un hadith stipulant qu'il ne doit y avoir « ni préjudice contre soi ni action nuisible à autrui " (la darar wa lâ dirâr) - où donc l'intérêt propre est pris en compte dans la limite des torts suceptibles d'être portés à autrui. Le différend opposant le fiqh inspiré des anciens maitres (salafi) à al-Tûfî se ramenait dès lors à la primauté accordée par ce dernier à l'intérêt: constituant pour lui l'un des premiers fondements de la sharia, il en représente l'intention primordiale, alors que pour les fuqahâ' il n'en est qu'une parmi d'autres.

Le faqîh égyptien al-cIzz bin cAbd al-Salâm $(\mathrm{m} .660 \mathrm{H})$ avait pourtant été plus loin dans son exploration de la notion d'intérêt qu'il disposait selon trois niveaux : la nécessité, le besoin et la complétude. «Sa contribution est l'un des points les plus lumineux dans la jurisprudence islamique, qu'il éloigne des tergiversations théoriques pour l'ancrer dans 
la vie. Le fait que son apport n'ait pas eu d'incidence réelle dénote la rupture qui s'était installée entre le fiqh et la vie. »

Al-Shâtibî (m. 790 H) marque un autre recul par rapport à al-Tûfî en déterminant les cinq intentions de la Loi (maqâsid al-sharî́a) : sauvegarde de la religion (hifz al-dîn), de la personne (hifz al-nafs), de l'entendement (hifz al-caql), de la progéniture (hifz al-nasl) et enfin des biens (hifz al-mâl). La notion d'» intention » diffère de celle d'» intérêt » en ce sens qu'elle ne fait pas jouer l'autonomie du faqîh, sans compter les multiples adaptations et réductions qui lui ont été imposées. L'auteur rappelle à ce propos qu'aujourd'hui encore on ne mentionne, comme moyen de sauvegarde de l'entendement, que la prohibition de l'alcool. Sur ce point, n'aurait-il pas mieux valu débattre par exemple des problèmes de la science, de l'acquisition du savoir et du développement des méthodes d'enseignement ? Et pour la sauvegarde des biens, au lieu de n'y voir que l'autorisation de la vente et l'interdiction de l'usure, n'aurait-on pu s'attendre à une caractérisation de la propriété privée par sa fonction productive et sociale...?

L'apport fondateur à tous les niveaux, estime l'auteur, fut celui de Shâh Walî-Allâh de Delhi (m. $1176 \mathrm{H})$, qui oriente la connaissance islamique vers une compréhension pénétrante et sensible des intentions de la sharia et de l'intérêt en forgeant le concept de « correspondances » (irtifâqât).

Critiquant al-Tûfî pour son appréciation étroite des préceptes relatifs au culte (interprété comme la manifestation mécanique d'une obéissance aveugle à Allâh), Shâh Walî-Allâh affirme l'importance de l'intégration du culte au domaine de la connaissance car "les indices et approches multiples de la science procurent la paix du cœur", indiquait-il, faisant allusion au mot d'Ibrâhîm (Abraham) dans le Coran. C'est que, affirme Gamâl al-Bannâ, les préceptes relatifs au culte comme les statuts juridiques « ne sont point des pierres sourdes qui séquestrent leur sens ", mais, comme le montre Shâh Walî-Allâh, "ce sont des entités vivantes qui oscillent en permanence entre le but prévu par le Législateur et l'attention due aux humains à qui elles ont été révélées, à leur époque, à leur situation, aux circonstances de leur quotidien avec lesquelles elles concordent et correspondent... »"

L'auteur relève la ressemblance entre le faqîh de l'Inde et Ibn Khaldûn dans la richesse des significations que l'on retrouve dans l'ouvrage longuement cité du premier Hujjatu-llâhi-l bâligha (La Raison suprême de Dieu) -, dans le rôle qu'il accorde aux structures sociales; enfin dans cette profonde vision philosophique qui pénètre "les secrets de la législation ». Cette vision, poursuit-il " est une clé nécessaire si l'on veut établir les fondements de la jurisprudence sur une base solide, en correspondance avec ces secrets et avec leur sens intime».

La méconnaissance des « limites » fixées par Dieu. Le deuxième concept conflictuel auquel s'attaque l'auteur est celui de hudûd (s. hadd) - signifiant littéralement "frontières " ou "limites" fixées par Dieu (hudûd Allâh) - dont on relève près de quatorze occurences dans le Coran dans des versets comportant des orientations générales ou des directives concernant l'héritage, les droits de la femme, le jeûne, le système économique et politique mais ne faisant nulle allusion aux "peines légales ", malencontreusement assimilées au mot hudûd. «L'expression hudûd Allâh est liée à une idée fondamentale de la sharia, à l'esprit et à la philosophie de l'islam, à savoir, le rapport entre ce qui est autorisé ou ne l'est pas et qui n'est nullement réductible aux peines prescrites » : l'auteur conteste par ailleurs l'association directe faite par certains 
entre hudûd et "ce que Dieu a révélé " (ma anzala-llâh). Cette dernière expression est exhaustive et désigne le Livre révélé en tout ce qu'il comporte, qu'il ait pour nom Torah, Évangile ou Coran, et si l'on comptait le nombre de versets où elle apparaît aux côtés du mot « droit » (al-haqq), on comprendrait que l'essentiel est dans la conjonction de la révélation et de la justice.

Par ces mises au point terminologiques, l'auteur nous donne à mesurer la distance que le figh a prise avec sa source principale et met en garde contre les distorsions réduisant l'islam aux peines légales plus ou moins bien comprises. C'est pourquoi il revient au sens usuel du mot hudûd ou "peines légales" faisant remarquer que celles-ci constituent l'infime partie du droit pénal islamique en comparaison des peines laissées à l'appréciation du juge ou des responsables politiques (taczîr, pl. tacâzîr), sachant que ce droit pénal ne constitue à son tour qu'une partie de l'ensemble des lois du droit islamique public ou privé ou de la sharia, laquelle n'est elle-même qu'une des composantes de l'islam.

69 En effet, constate l'auteur, seuls cinq versets sur les plus de six mille deux cents qui composent le Coran, déterminent des peines en cas de vol, d'adultère, de calomnie, d'acte de guerre et de crime - encore que certaines peuvent être abolies en cas de repentir -, et ne mentionnent pas de détails quant au mode d'exécution. Par ailleurs, fait-il remarquer, il n'est fait allusion à aucune sanction punitive contre la boisson d'alcool ou contre le reniement de la foi (al-ridda). À côté des milliers de versets relatifs à la piété (taqwa), au pardon (istighfâr), au repentir (tawba), le nombre excessivement restreint des préceptes pénaux proprement dits est le signe que le Coran privilégie la foi (caqîda) par rapport à la Loi (sharî́a) et que sans amoindrir l'importance des préceptes légaux nécessaires à la vie de toute société, il donne à comprendre que «la mission d'ouverture à la connaissance, à la justice et à la liberté ne peut s'accomplir que dans un climat de profonde croyance ».

Du texte coranique émane donc le cadre théorique du crime et du châtiment en islam. Le Coran configure, autant par le fond que par la forme, la dialectique qui met dans un rapport d'interaction et d'inséparabilité deux entités différentes, non contradictoires, complémentaires, la foi et la Loi, ayant chacune sa nature, son domaine et ses mécanismes propres, l'une agissant directement sur l'individu par la persuasion, l'exhortation au bien, tirant son argument suprême du Coran, l'autre visant prioritairement la société et opérant par dissuasion avec pour raison le pouvoir.

71 L'auteur étudie, dans ce contexte, l'incidence de la foi sur la pratique juridique au temps du Prophète, d'abord en donnant à méditer : l'attitude de ses contemporains qui ont vu dans la peine légale un moyen expiatoire et de purification ; les clauses exigeant l'aveu du coupable comme pièce à conviction maîtresse et prévoyant aussi sa rétractation; enfin, le conseil du Prophète recommandant d'éviter d'en arriver aux peines légales (tacâfû-l hudûd) et "la continence» dont il faisait preuve dans l'application de celles-ci allant jusqu'à souffler à l'accusé de nier, et instituer ainsi un principe général enjoignant de "repousser les peines en cas d'incertitude " (idra'u-l hudûd bi-l shubuhât, principe pouvant englober de très nombreux cas). Au point qu'Ibn Hazm a mis en doute l'authenticité du hadith qui en témoigne, y voyant une manœuvre pour abandonner les peines légales. «Exagération!, commente l'auteur, dans la mesure où le but, en définitive, est de prouver l'accusation. »

72 Si cette exemplarité « utopique » est impossible à réaliser dans sa forme initiale, elle n'a pas pour autant un caractère d'exclusivité et fonde une norme en comparaison de 
laquelle apparaît la pauvreté du fiqh moderne et, surtout, contemporain, dont les ouvrages ne mentionnent même pas le fait que le repentir œuvre en faveur de l'exemption des peines, voire « à transformer les fautes en actes de bienfaisance (Coran, $25,70) »$.

La Loi, par contre, est investie de la tâche de «réformer la société pour garantir la réforme de l'individu» et, à ce titre, elle ne peut rien négliger; la fermeté et l'objectivité sont ses armes. Toutefois, «la société islamique forme un tout et l'on doit pouvoir sentir dans ses moindres recoins, battre le pouls de son âme collective incarnée dans la justice et dont les ordres et interdits ne sont point contradictoires mais concordants ". C'est pourquoi, indique-t-il, on ne peut punir le voleur que s'il a été procédé à une redistribution des richesses par le biais de l'aumône légale purificatrice (zakât)". Aussi "ne convient-il pas de dévaloriser la réforme introduite par la Loi », poursuit-il, citant entre autres William Temple, l'ancien évêque de York", car celle-ci peut « créer un climat favorable à l'éclosion des valeurs morales ».

L'auteur analyse sous tous leurs aspects les diverses sanctions prescrites par la sharia, en particulier celle relative au vol, en les comparant aux lois du droit positif dans plusieurs pays et expose longuement le point de vue de fuqahâ' et hommes de loi contemporains qui ont contribué à faire revivre l'esprit du Coran dans le cadre précis de leur interprétation adéquate des prescriptions juridiques ou par la reconnaissance de leur opportunité, tels Cheikh 'Abd al-Mitcâl al-Sacîlî, Fâris al-Khûrî, Macrûf alDawalîbî, Muhammad Shahrûr, cAbdallâh al-cAlaylî...

Les trois composantes caractéristiques du fiqh. L'auteur clôt enfin cette partie d'analyse critique sur un point de la plus haute importance qui éclaircit, en outre, sa position quant au statut du texte et à son rôle sur le plan juridique. En étudiant le développement de la pratique du droit islamique ou jurisprudence au cours de l'Histoire, il dégage trois composantes qui ont profondément marqué et caractérisent, aujourd'hui encore, le fiqh: son aspect fragmentaire et l'hégémonie des études de langue et de la logique formelle.

Historiquement, explique l'auteur, la fragmentation a naturellement déterminé une jurisprudence naissante sollicitée par des « cas » isolés et « qui a évolué dans le souci de trouver des solutions juridiques propices et non dans celui d'établir une législation (tashrî́)».

77 Les fondements (usûl) du figh établis plus tard, ont eux-mêmes gardé ce trait en ne proposant qu'une méthode de déduction des statuts juridiques à partir du Coran et de la sunna, du raisonnement analogique (qiyâs) et du consensus (ijmâc), ces deux fondements secondaires devant fonctionner dans un rapport d'étroite dépendance à l'égard des deux premiers. Ceci, note l'auteur, est une conséquence de ce que le Coran, en particulier, a été considéré par les anciens comme la source des sentences et des jugements alors qu'ils auraient dû y voir la source d'une législation fondée sur la raison et l'intérêt public.

78 Le processus historique de démarquage du territoire du figh est à ce titre très significatif. Sans entrer dans les détails techniques de cette opération exposés par l'auteur, on retiendra la mise à l'écart, par les fuqahâ' traditionnels, des statuts et solutions juridiques non tirés des préceptes révélés mais inspirés par la raison (al-caql), des statuts théoriques relatifs à la foi (cf. vol.1), etc. 
79 Avec la complexité croissante accompagnant le brassage de sociétés diverses, le fiqh, pour lequel al-Shâficî proposait de nouveaux fondements auxquels «se rendit » la communauté - non sans retour, car Abû Hanîfa proposait aussi ses fondements essentiellement liés aux ramifications (furûc) et à la jurisprudence des tribunaux (fiqh alqadâ') -, garda ce trait spécifique. En effet, bien que n'opérant plus à partir de cas isolés (figh al-hâlât), la jurisprudence n'arrivait pas pour autant à se donner une base transcendante et continua à chercher des solutions juridiques dans les implications (cilla, pl. cilal) des préceptes coraniques (figh al-cilal) sans dépasser la lettre du texte afin d'en tirer la morale (hikma, pl. hikam). Ainsi, commente l'auteur, le Coran ayant autorisé le voyageur à rompre le jeûne du Ramadan, on s'en tient traditionnellement au texte sans réfléchir au fait que le $\mathrm{XX}^{\mathrm{e}}$ siècle offre à ce voyageur des moyens de transports autrement plus confortables; de même laisse-t-on un ouvrier qui jeûne suer sang et eau devant une forge sans le faire bénéficier du même précepte, qui vaut cependant davantage pour lui et pour ses semblables parce qu'il relève en premier lieu d'un sens profond, celui de la compassion et de la miséricorde divine.

La tentative d'établir des règles (qawâcid) pour le figh regroupant des lignes directrices ne parvint par suite qu'à consacrer sa fragmentation. Et dans ce contexte, l'effort incomplet d'al-Shâtibî qui, dans son interprétation partielle restait très en deçà des réelles intentions de la sharia, ne put opérer de percée fondamentale.

81 Pour pallier cet échec, il aurait fallu, indique l'auteur, "partir du même point que le Coran », le prendre dans sa totalité - parce que c'est ainsi et non dans ses parties isolées qu'il constitue le véritable livre de la Loi - et considérer «les principales valeurs morales (al-qiyam al-ra'îsiyya) » qu'il établit, de sorte que chacune d'elles devienne une source génératrice de statuts dont l'assise unificatrice serait le Coran.

82 Par ailleurs, sans nier l'apport des études de langue au fiqh impliqué dans l'action juridique - la responsabilité des fuqahâ' étant ici tributaire de leur compréhension de la syntaxe particulière du Coran et de la maîtrise de son vocabulaire -, l'auteur conteste la ratiocination grammaticale et lexicographique sur laquelle s'est greffée la logique formelle pour "neutraliser l'aptitude des fuqahâ' à statuer », allant jusqu'à "vider de son sens une idée aussi importante que celle des intentions de la sharia ». Il s'élève en outre contre l'envoûtement réducteur exercé par la logique aristotélicienne depuis le premier siècle de l'hégire sur les fuqahâ" qui ont fini par «sacrifier la vérité à des formules abstraites ".

Les fondements de la loi

83 La raison. Poursuivant sa lecture critique du patrimoine jurisprudentiel et islamique, l'auteur approfondit les approches et notions étudiées dans le premier volume et propose dans la seconde partie du présent ouvrage de nouveaux fondements de la Loi (usûl al-sharîa $a$ ) dictés par la raison, elle-même établie comme fondement premier et principe primordial pour reconnaître l'existence de l'Être suprême et comprendre le Coran.

Or celui-ci, par la mise en œuvre simultanée du cœur (al-qalb) et de la raison (al-caql), institue une logique qui ouvre pour le moins une double perspective : en convainquant la raison humaine d'intégrer ses limites «naturelles » devant l'inconcevable - dont l'approche est réservée au cœur, réceptacle de la foi (al-îmân) -, elle l'oriente par ailleurs vers le champ illimité de la connaissance où se développe son intelligence du bien et du mal (al-tahsîn wa-l-taqbîh) et de la justice (al-cadl), « fruit de la sharia ». 

fondement de la sharia, l'auteur rappelle que le Livre, « espace ouvert au multiple et à l'alternative ", dont chaque verset renaît à un sens nouveau à travers les époques et les lieux, est en soi «le miracle de l'islam». Par ailleurs, indique-t-il, «le Coran ne s'apparente nullement à un code de lois où les juges puiseraient leurs sentences »; livre 
de guidance au premier degré, il donne à considérer l'Être suprême, «symbole et principe originel de toutes les valeurs ", à travers sa création et dans le dit de ses Noms/Attributs, desquels procèdent les notions du Droit (al-haqq) et de l'éthique dans leur absolue perfection.

La rareté des statuts juridiques, leur formulation générale - exception faite du verset sur la dette ajournée et d'indications relatives à l'héritage -, suscitent la réflexion à laquelle les valeurs juridiques servent de «boussole». La double erreur de la jurisprudence islamique, estime l'auteur, fut d'abord de voir dans le Coran un livre de lois, alors qu'elle aurait dû partir de son enseignement moral pour fonder les statuts et les garantir ; ensuite « de croire que, sans restriction, la sunna était habilitée à pallier le manque de précision intentionnel du Coran, alors qu'elle devait être lue, filtrée, à travers la grille des valeurs posées par le Livre et non le contraire».

On constate « les conséquences de cette erreur dans le nombre important de statuts qui démentent l'esprit de l'islam et (...) contribuent à sa décadence ", conclut l'auteur avant d'exposer « les valeurs dominantes » susceptibles de générer des statuts adéquats. Ces dernières sont distinguées des nombreuses valeurs morales complémentaires en ce sens que celles-ci ne peuvent être à l'origine d'un statut légal, mais s'assortissent aux premières pour en donner la parfaite mesure.

Le couple Droit/justice (al-haqq/al-cadl) se partage les territoires de la foi (caqîda) et de la Loi (sharîa) et constitue la clef de voûte des valeurs dominantes. Le Droit est l'attribut de Dieu par excellence" dont "la nature théorique et objective » n'a pas son corollaire dans les autres systèmes juridiques. Ces derniers "procèdent en effet de l'homme et renvoient aux droits respectifs des diverses parties " suppléant à leur caractère subjectif par le concept de « droit naturel ».

95 La justice étant la mise en pratique du Droit, l'islam en a fait le fondement et la condition de crédibilité des statuts légaux devant inspirer toutes les composantes de la Loi dans les domaines politique, économique et social. Par ailleurs, le caractère relatif de la justice eu égard au Droit (attribut absolu de Dieu) tend à s'estomper à la lumière des particularités qui définissent chaque situation: la compassion ( $r a h m a)$, le pardon (al-cafw, al-istighfâr), sont autant de valeurs complémentaires citées par le Coran qui viennent «adoucir » la justice, de même que le repentir (tawba) doit pouvoir infléchir sa sentence (cf. plus haut al-hudûd), un des attributs de Dieu étant «Celui qui agrée le repentir (al-tawwâb)».

96 Un code des statuts légaux islamiques devrait consacrer une de ses branches au repentir, en expliquer les mécanismes et en étudier les conséquences autant sur les statuts que sur la vie sociale, indique l'auteur qui salue à ce propos l'initiative de Nelson Mandela et Desmond Tutu en Afrique du Sud, laquelle a donné l'exemple de ce que peut être un tribunal acceptant les aveux repentants d'un pan entier de la société - tribunal devant lequel ont comparu les partisans de l'ancien régime de l'apartheid, pour exprimer leurs regrets et proclamer leur repentir sans que châtiment s'ensuive - et l'incidence d'une pareille action sur la réforme individuelle et sociale.

97 La liberté de pensée et de croyance (hurriyatu-l-fikr wa-l-ictiqâd) - garantie de la foi et parant à l'ingérence d'un quelconque pouvoir -, la latitude facilitant l'action (al-taysîr) et l'indulgence (al-samâha), se conjuguent dans l'organisation d'une vie sociale où sont respectées la dignité de l'homme et la diversité voulue par Dieu, de même que, sur le plan économique, le déboursement ou dépense (al-infâq) - principe sans cesse 
encouragé par le Coran qui stigmatise l'avarice -, sera pris pour base des statuts régissant les tractations financières, interdisant l'accaparement des terres pour une durée indéfinie, etc.

La sunna. L'existence de valeurs morales qui font éclore la conscience humaine, les lois sociales et l'existence de chefs qui les personnifient et les enracinent dans les esprits sont les deux conditions préalables à la réforme sociale, "Dieu ayant symbolisé les valeurs morales par le Coran et le chef qui œuvre à les expliciter et à mettre en pratique les lois qui les traduisent dans la société par le Prophète ». L'auteur résume l'argumentation développée dans le deuxième volume et en tire la conclusion : la sunna ajustée au Coran - et non le contraire - et délivrée des "camisoles de force " dans lesquelles s'est enfermé le figh, apparaît donc comme le troisième fondement de la nouvelle jurisprudence, moteur qui précise et combine dans la syntaxe de la vie les paradigmes énoncés par le Coran.

99 La coutume. Enfin, le quatrième fondement établi par l'auteur est la coutume (al-curf) en lieu et place du consensus ou accord unanime (ijmâc) des croyants ou des ulémas, mis en doute ou jugé impossible par de nombreux fuqahầ tels Ibn Hanbal (qui a été jusqu'à dire : «Celui qui prétend qu'il y a consensus est un menteur »!), Ibn Hazm, al-Shâficî, Ibn al-Wazîr... "À supposer que pareil accord fut possible, indique l'auteur, cela n'exclut pas l'erreur de jugement: en constitue un exemple, l'accord des fuqahâ' concernant l'abrogation (naskh) de versets du Coran ou le châtiment du renégat ; dans les deux cas, l'écart est évident dans la mesure où ils se réclament d'arguments subjectifs aux dépens du fondement général (...). Quant à l'insistance des contemporains à considérer l'ijmâa comme une vérité incontournable alors qu'il n'a en fait jamais existé, elle dénonce leur tendance à ruminer le passé et leur démission devant la pensée rationnelle. »

Si le rôle de l'ijma $\hat{a}^{c}$ était d'introduire une dimension humaine dans le rapport juridique et prétendait ouvrir l'accès à une participation maximale (qui s'est avérée illusoire), la coutume, généralement considérée comme un fondement subsidiaire, paraît avoir un apport plus essentiel à ce niveau car " en permettant au temps de respirer $»^{18}$, elle ouvre la voie royale à la participation sociale spontanée ou rationnelle.

Outre le fait que des indices du Coran et de la sunna autorisent à voir dans la coutume une source de juridiction, indique l'auteur, celle-ci constitue le terrain de l'humain à part entière où le principe, sauf en de rares exceptions, est de ne point interdire et où il n'apparaît de contradiction fondamentale [avec le bien] que dans le cas d'une totale inversion des valeurs. Toutefois, constate l'auteur, la jurisprudence traditionnelle n'a retenu la coutume que dans une perspective traditionnelle, transformant ce terrain de l'humain en un goulot d'étranglement où se bousculent le plus grand nombre de contraintes et de prohibitions, concernant la femme en particulier.

Il n'en demeure pas moins qu'étant « la voix du temps invincible dont nulle force ne peut arrêter la progression ni les changements qui l'accompagnent, et le fruit de l'interaction des civilisations, des cultures et des peuples ", la coutume ouvre un champ privilégié à la réflexion sur les rapports du passé et du présent, du semblable et du différent, de la continuité et de la rupture imposée par l'évolution et que toute législation doit intégrer. "Peu importe, écrit l'auteur, si des coutumes semblent déroger à certains textes car les alternatives qu'offre la sharia assurent la souplesse nécessaire pour traiter avec l'irruption du nouveau et pour s'inspirer des plus hautes intentions de la Loi en les préférant à la lettre du texte... » 


\section{NOTES}

1.Gamâl Al-Bannâ, Nahwa Fiqh-in Gadîd (Vers une nouvelle jurisprudence islamique), vol.1, Le Caire, Dâr al-fikr al-islâmî, 1996, 207 p.

2.Il s'agit du hukm shari (pl. ahkâm : statut légal, solution juridique) qui est différent du hukm signifiant " gouvernement ».

3.Les sources fondatrices du figh telles que posées par les diverses écoles juridiques puis consacrées par la coutume sont : le Coran, la sunna ou tradition du Prophète, le raisonnement analogique (qiyâs assimilé à l'ijtihâd par l'imam al Shâficî) et le consensus (ijmâc). L'anarchie avait un moment régné parce que la tradition du Prophète n'était pas encore entièrement transcrite et que telle solution juridique qui se justifiait par une référence à tel hadith se trouvait bientôt contredite par un autre hadith connu plus tardivement, etc., ainsi que l'indique l'auteur, sachant que la transcription de la sunna n'a commencé qu'environ un siècle après la mort du Prophète et fut considérée comme le corollaire du Coran.

4.Gamâl Al-Bannâ, Nahwa figh-in gadîd : al-sunna (Vers une nouvelle jurisprudence islamique : la sunna), vol. 2, Le Caire, Dâr al-fikr al-islâmî, 1997, 279 p.

5. "Passage au tamis » que l'on doit notamment aux grands imams al-Bukhârî (194-256 H), Muslim (206-261 H), Abû Dâwûd (202-275 H), al-Turmuzî (209-279 H), al-Nisâ'î $(215303 \mathrm{H})$, Ahmad Ibn Hanbal (164-241 H).

6.Tawfîq Sidqî : médecin qui, dans la première décennie du siècle, écrivait régulièrement des articles dans la revue al-Manâr fondée par Rashîd Ridâ. Ahmad Amîn : diplômé de l'École de juridiction islamique (Madrasat al-qadâ' al-sharî), fut doyen de la faculté des lettres de l'université Fouad 1eret auteur de la trilogie historique, Fajr al-islâm (L'Aube de l'islam). Mahmûd Abû Rayyâ : auteur de Adwâ' cala al-sunna almuhammadiyya (Lumières sur la sunna de Muhammad, 1950), il fut le premier à remettre en cause Abû Hurayra, un des principaux transmetteurs du hadith. Ismâ̂l Mansûr : ancien membre d'al-Gamciyya al-shariyya, principale association d'inspiration traditionnelle (salafi), il a totalement changé d'orientation et a récemment publié l'ouvrage critique cité par l'auteur.

7.Ibrâhîm al-Wazîr, m. $800 \mathrm{H}$ : membre d'une famille yéménite de vieille souche et grand faqîh de l'école zaydite qui parmi les sectes chiites est la plus proche du sunnisme. 8.Doyen de la faculté de la sharia de Syrie dans les années soixante, il fut l'un des dirigeants du mouvement des Frères musulmans dans ce pays.

9.Doyen de la faculté de la sharia de Syrie dans les années cinquante et professeur d'université, il eut pour étudiant Mustafâ al-Sibâî.

10.Dans un ouvrage précédent Mâ Bacd al-Ikhwân al-muslimîn (Qu'y aura-t-il après les Frères musulmans... ?), l'auteur fait la distinction entre la notion de " force ", celle-ci étant nécessaire, et la notion de « violence » où prédomine l'arbitraire. Cf. également cette distinction chez Hannah Arendt, Du mensonge à la violence, Essais de politique contemporaine, Calmann-Lévy, Paris, 1972.

11.Le calife ‘umar avait montré que même le texte sacré, le Coran, appelait un effort d'interprétation personnel lorsqu'il s'était abstenu, en temps de famine, d'appliquer la peine destinée aux voleurs. Cet exemple est cité par l'auteur dans le premier volume, dans le chapitre consacré à l'ijtihâd. 
12.Gamâl al-Bannâ, Nahwa fiqh-in Gadîd (Vers une nouvelle jurisprudence islamique), vol.3., Dâr al-fikr al-islâmî, 1999, 312 p.

13.Cf. le récent ouvrage de l'auteur : Al-Mar'a al-muslima bayna tahrîr al-Qur'ân wa taqyîd al-fuqahâ' (La Femme musulmane entre la libération du Coran et les restrictions des jurisconsultes, 1998) qui est un prolongement de son travail d'examen critique de la tradition du Prophète telle que rapportée par les traditionnistes, dans le deuxième volume consacré à la sunna.

14. On pense ici à Jacques Berque qui, pour évoquer cet « absolu qui se communique à l'homme ", parle d'» éternité émigrée dans le temps » : Jacques Berque, Relire le Coran, Paris, Albin Michel, 1993, p. 52.

15.Le mot arabe zakât connote la purification, d'où « l'aumône purificatrice » dans la traduction de Berque.

16.Dans The Ethics of Penal Action, William Temple devenu plus tard archevêque de Canterbury fut le premier président du conseil de direction de l'Institut de la culture ouvrière de Grande-Bretagne. L'auteur évoque également l'initiative d'Eisenhower d'envoyer l'armée fédérale à Little Rock pour mettre fin à l'état de discrimination raciale contre les noirs, initiative qui eut pour effet de réduire de plusieurs années au moins leurs souffrances.

17.Dans Nadhariyyat al-cadl fi-l fikr al-ûrubbî wa-l- fikr al-islâmî (La théorie de la justice dans la pensée européenne et dans la pensée islamique, 1995) l'auteur étudie la dimension polysémique du mot dont on ne retrouve pas le sens dans les langues européennes où il n'existe pas de synonyme précis au terme coranique al-haqq dans son abstraction absolue. Dans la plupart de ces langues, écrit-il, le mot « droit » s'accompagne de la préposition « de » : «droit de propriété », « droit de vote »... Le mot peut signifier « juste » (right) contraire de « faux », etc.

18.L'expression est de Tâj al-Dîn al-Subkî (VIII siècle de l'hégire) ; elle est mise en tête du chapitre par l'auteur à la suite du verset : « Pratique le pardon ( $a f w)$, ordonne ce qui est reconnu pour être le bien (urf), écarte-toi des ignorants» (Coran, 7, 199).

INDEX

Mots-clés : al-Banna (Gamal), Coran, exégèse coranique, interprétation, islam, liberté de religion, religion 